\title{
THE BIOCHEMICAL AND MORPHOLOGICAL DEVELOPMENT OF DIFFERENTIATING DOPAMINE NEURONS CO-AGGREGATED WITH THEIR TARGET CELLS OF THE CORPUS STRIATUM IN VITRO ${ }^{1}$
}

\author{
CONNIE KOTAKE, ${ }^{2}$ PHILIP C. HOFFMANN, AND ALFRED HELLER
}

Department of Pharmacological and Physiological Sciences, The University of Chicago, Chicago, Illinois 60637

Received January 4, 1982; Revised March 15, 1982; Accepted March 22, 1982

\begin{abstract}
Dopamine neurons from the rostral mesencephalic tegmentum of embryonic mouse brain were dissociated and allowed to co-aggregate in vitro with dissociated cells from the embryonic corpus striatum. The differentiation of dopamine neurons in this system was characterized by examining the developmental course of a number of indices of dopaminergic function. Fluorescent cell bodies and patches of punctate pericellular fluorescence due to endogenous dopamine were visualized in the co-aggregates after 14 and 21 days in culture. The punctate pericellular fluorescent pattern resembled that seen in the intact neostriatum. Punctate pericellular fluorescence could be detected in 3- and 7-day co-aggregates only if they were exposed to $1 \mu \mathrm{M}$ dopamine. The density of the pericellular fluorescence increased with time in culture. Endogenous dopamine levels also increased with time in culture, reaching maximal levels of $22 \mathrm{ng} / \mathrm{mg}$ of protein at 21 days. The accumulation and retention of exogenous dopamine followed a developmental course similar to that observed for endogenous dopamine content. At 21 days in culture, the co-aggregates accumulated 11 to 13 times as much dopamine as was accumulated by 3-day cultures. The sensitivity to reserpine and 6hydroxydopamine also was examined. Co-aggregates cultured for 7,14 , or 21 days and pretreated with reserpine $\left(10^{-7} \mathrm{M}\right)$ for $24 \mathrm{hr}$ were able to accumulate $54 \%, 5 \%$, and $6 \%$ as much dopamine as untreated cultures, respectively. 6-Hydroxydopamine $\left(10^{-4} \mathbf{M}\right)$ pretreatment produced a similar agedependent decrease in the amount of dopamine accumulated. Such co-aggregates cultured for 7, 14, and 21 days accumulated $41 \%, 10 \%$, and $7 \%$ as much dopamine as control cultures, respectively. No significant effect of either reserpine or 6-hydroxydopamine pretreatment was observed in 3-day cultures. Dopamine neuron-containing co-aggregates show an age-dependent development of mechanisms for the synthesis, accumulation, and storage of dopamine and are able to elaborate and maintain axons and terminals in culture. Thus, the dopaminergic neurons in these co-aggregates display characteristics of anatomical, biochemical, and pharmacological development similar to those seen in intact brain.
\end{abstract}

Dopaminergic neurons are important determinants of motor, ingestive, emotional, and neuroendocrine functions in animals, including man (Seiden and Dykstra, 1977). Studies on the anatomical and biochemical development of central dopaminergic systems represent an important approach to the understanding of the func-

\footnotetext{
${ }^{1}$ This research was supported by National Institute of Mental Health Grant MH 28942. These data have been presented in preliminary form at the Society for Neuroscience Annual Meeting, October 1981 (Kotake, C., P. C. Hoffmann, and A. Heller (1981) Soc. Neurosci. Abstr. 7: 847). We thank Eligia Dimapilis and Francis Karapas for technical assistance and Dr. T. G. Heffner for critical comments.

${ }^{2}$ To whom correspondence should be addressed at Department of Pharmacological and Physiological Sciences, The University of Chicago, 947 East 58th Street, Chicago, IL 60637.
}

tional role of dopaminergic neurons in these processes. The ontogeny of central dopaminergic neurons in vivo has been studied in some detail (Coyle, 1974; Coyle and Campochiaro, 1976; Golden, 1973; Lauder and Bloom, 1974; Olson and Seiger, 1972; Porcher and Heller, 1972; Seiger and Olson, 1973). However, the complexity of neuronal organization in the intact developing fetal brain as well as difficulties in experimentally manipulating these systems during this time period represent formidable barriers to characterization of the mechanisms which direct this development.

Dopaminergic neurons arising in the substantia nigra and ventral tegmental area of the rostral mesencephalic tegmentum (RMT) innervate a number of structures in the mammalian forebrain, including the corpus striatum (CS) (Moore and Bloom, 1978; Ungerstedt, 1971). Re- 
cently, it has been demonstrated that the RMT from the embryonic brain can be removed, dissociated into single cells, and reaggregated in a rotatory culture system with dissociated cells from the embryonic CS (Hemmendinger et al., 1981). After 7 days in culture and exposure to exogenous dopamine, the resulting co-aggregates exhibited intense, punctate pericellular fluorescence which closely resembled the appearance of the dopaminergic, axonal fluorescence patterns observed in the intact, adult corpus striatum.

'The fact that significant histofluorescence could not be observed in the absence of exposure to exogenous dopamine to load the dopamine neurons suggested that the development of these neurons within the co-aggregates may not be complete by 7 days in culture. Therefore, the present investigation was undertaken to characterize the development of the dopamine neurons in RMT-CS co-aggregates by assessing a number of indices of dopaminergic function after $3,7,14$, or 21 days in culture. Histofluorescence derived from endogenous dopamine was examined and the amounts of dopamine as well as tyrosine hydroxylase activity were measured in such co-aggregates. In addition, the ability of these cultured dopaminergic neurons to accumulate and retain exogenous dopamine and the sensitivity of these processes to reserpine and 6-hydroxydopamine (6OHDA) were examined as a function of time in culture. The results of these experiments demonstrate an agedependent development of the mechanisms for accumulation, synthesis, and storage of dopamine in such coaggregates which is similar to that seen in developing animals. The normal development of dopamine neurons in this in vitro system should allow one to explore the factors involved in this development since it is possible to control both the fluid and cellular environment of the differentiating dopaminergic cells.

\section{Materials and Methods}

Preparation of RMT-CS co-aggregates. RMT-CS coaggregates were prepared as previously described (Hemmendinger et al., 1981). Briefly, RM'T and CS were obtained from the brains of mouse embryos on the 14th day of gestation. The RMT, containing the dopaminergic neurons, was dissected in accordance with its histofluorescent localization in mouse embryos of this age (Golden, 1973). CS was obtained from the cerebral lobes following removal of the septum and surrounding cortex. The tissue then was dissociated after incubation with $0.67 \%$ trypsin and allowed to reaggregate as previously described (Garber and Moscona, 1972; Hemmendinger et al., 1981). Co-aggregates were prepared using $5 \times 10^{6}$ cells of each tissue type.

Fluorescence histochemistry. Co-aggregates were maintained in culture for $3,7,14$, or 21 days. To examine endogenous histofluorescence, the co-aggregates were washed three times in $1 \mathrm{ml}$ of Tyrode's solution. After removal of the excess solution, the co-aggregates were frozen in a mixture of $95 \%$ liquid propane and $5 \%$ propylene cooled in liquid nitrogen and processed by the Falck-Hillarp histofluorescence method (Falck et al., 1962). For examination of dopamine histofluorescence following loading with exogenous dopamine, the co-aggregates were incubated with $1 \mu \mathrm{M}$ dopamine in $1 \mathrm{~mm}$ ascorbic acid for $10 \mathrm{~min}$ at $35^{\circ} \mathrm{C}$. In some cases, the coaggregates were pretreated with either $10^{-7} \mathrm{M}$ reserpine, $10^{-4}$ M 6-OHDA, or their respective vehicles $24 \mathrm{hr}$ prior to loading with exogenous dopamine. The co-aggregates then were washed in Tyrode's solution and processed for histofluorescence as described above. Paraffin sections $(10 \mu \mathrm{m})$ were mounted in paraffin oil and viewed with a Leitz Orthoplan microscope equipped with a 200 -W mercury lamp under conditions for the visualization of the dopamine fluorophore. Fluorescent sections were photographed through a $\times 10$ or $\times 25$ objective on Kodak TriX or Ektachrome 400 film. All photomicrographs were obtained with the identical time of exposure ( $30 \mathrm{sec})$.

High performance liquid chromatography (HPLC) analysis of biogenic amine content. For the determination of endogenous amine levels, co-aggregates were washed three times with $1 \mathrm{ml}$ of Tyrode's solution and the tissue pellets were frozen in liquid nitrogen. The amount of exogenous dopamine accumulated and retained and the sensitivity of the process to reserpine and 6 -OHDA were determined by incubating co-aggregates with either 6-OHDA, reserpine, or their respective vehicles after $2,6,13$, or 20 days in culture. The final concentrations of 6-OHDA and reserpine were $10^{-4}$ and $10^{-7} \mathrm{M}$, respectively. In addition, pargyline $(10 \mu \mathrm{M})$ was added to flasks containing 6-OHDA or its vehicle. Drug-containing media were replaced with fresh drug-free media $1 \mathrm{hr}$ later. Twenty-four hours later, $1 \mu \mathrm{M}$ dopamine in $1 \mathrm{~mm}$ ascorbic acid was added and the flasks were incubated for $10 \mathrm{~min}$ at $35^{\circ} \mathrm{C}$. The co-aggregates then were washed three times with $1 \mathrm{ml}$ of Tyrode's solution and placed in Erlenmeyer flasks containing $3.5 \mathrm{ml}$ of tissue culture media (Eagle's minimal medium (Microbiological Associates) containing $8.7 \%$ horse serum (Gibco)) placed in a rotary incubator shaker (New Brunswick) at $90 \mathrm{rpm}$ and incubated for 30 or $60 \mathrm{~min}$ at $35^{\circ} \mathrm{C}$. Preliminary experiments using $\left[{ }^{3} \mathrm{H}\right]$ dopamine revealed that these incubation times were sufficient to remove the $\left[{ }^{3} \mathrm{H}\right]$ dopamine taken up into aggregates of CS cells alone, which do not contain dopamine neurons. The co-aggregates then were collected by centrifugation at $200 \times g$ for $2 \mathrm{~min}$ and washed twice with $1 \mathrm{ml}$ of Tyrode's solution, and the pellets were frozen in liquid nitrogen. Tissue was stored at $-80^{\circ} \mathrm{C}$ until HPLC analysis. Prior to chromatography, the tissue was homogenized for $10 \mathrm{sec}$ in $100 \mu \mathrm{l}$ of $0.4 \mathrm{~N}$ perchloric acid with an ultrasonic tissue disrupter (Branson). The resulting homogenate was centrifuged at 10,000 $\times g$ for 10 min. Twenty milligrams of alumina was added to the supernatant and the amines were eluted with 200 $\mu \mathrm{l}$ of $1 \mathrm{~mm}$ acetic acid. Protein in the perchloric acidprecipitable material was determined by the method of Lowry et al. (1951) using bovine serum albumin as the standard. The amine content in the acetic acid eluate was analyzed by HPLC. The basic chromatographic system consisted of a Milton Roy minipump (model 396), a reverse phase column (Altech RSil C18 HL), an amperometric detector (BAS LC2A) and injector valve with a 100- $\mu$ l sample loop, a carbon paste electrode (BAS), and a time base chart recorder (Linear). The mobile phase consisted of a citrate/phosphate buffer (110:100 mM, pH 2.5) containing EDTA $(0.1 \mathrm{mg} / \mathrm{ml})$ and octyl sodium sulfate $(0.03 \mathrm{mg} / \mathrm{ml})$. The flow rate was maintained at 1 $\mathrm{ml} / \mathrm{min}$. The carbon paste electrode potential was +0.72 
$\mathrm{V}$ versus the silver chloride reference electrode. Standards were prepared in $1 \mathrm{~mm}$ acetic acid.

Tyrosine hydroxylase activity. Co-aggregates were washed three times with $1 \mathrm{ml}$ of Tyrode's solution and homogenized in $500 \mu \mathrm{l}$ of $50 \mathrm{~mm} 2-(N$-morpholino)ethanesulfonic acid (MES) buffer, $\mathrm{pH}$ 6.0, containing $0.2 \%$ Triton $\mathrm{X}-100$. The homogenates then were centrifuged at $10,000 \times g$ for $10 \mathrm{~min}$ and the supernatant was used as the source of tyrosine hydroxylase. Incubations were carried out for $30 \mathrm{~min}$ at $37^{\circ} \mathrm{C}$ in a buffer containing $\mathrm{Fe}\left(\mathrm{NH}_{4}\right)_{2} \mathrm{SO}_{4},(40 \mu \mathrm{g} / \mathrm{ml})$, the dopa decarboxylase inhibitor NSD-1055 $(0.2 \mathrm{mg} / \mathrm{ml})$, mercaptoethanol $(30 \mu \mathrm{g} / \mathrm{ml})$, tyrosine (0.2 mM), DL-6-methyl-5,6,7,8-tetrahydropterine $(6.6 \mathrm{mM})$, and $50 \mu \mathrm{l}$ of tissue homogenate containing 100 to $200 \mu \mathrm{g}$ of protein. The reaction was terminated by addition of $400 \mu \mathrm{l}$ of $0.5 \mathrm{~N}$ perchloric acid. The assay tubes then were centrifuged and protein was determined on the pellets. The supernatants were purified on alumina (60 to $70 \%$ recovery) and the dopa content in the alumina eluate was analyzed by HPLC as described above.

Statistics. The significance of differences between data groups was assessed using the Student's $t$ test (two tailed).

\section{Results}

Development of endogenous dopamine histofluorescence. Dopamine neurons were not visualized by the
Falck-Hillarp histofluorescence procedure in RMT-CS co-aggregates that had been cultured for 3 or 7 days. However, co-aggregates maintained in culture for 14 days exhibited patches of bright green fluorescence characteristic of dopamine-containing axons (Fig. 1A). Larger and more prominent patches of punctate fluorescence were observed in co-aggregates maintained in culture for 21 days (Fig. $1 B$ ). This fluorescence appeared to be pericellular in nature, surrounding nonfluorescent areas of approximately $10 \mu \mathrm{m}$ in diameter. In addition, numerous brightly fluorescent cell bodies also were observed in this material (Fig. 1B). These cells measured 15 to $20 \mu \mathrm{m}$ in diameter and were typically ovoid and multipolar.

Development of endogenous dopamine levels. The levels of dopamine in RMT-CS co-aggregates increased progressively with time in culture (Fig. 2). Levels of dopamine were relatively low at 3 and 7 days in culture but displayed rapid increases between 7 and 21 days in culture. During the period between 7 and 14 days, there was a 4.5-fold increase in dopamine levels, with a further increase of 4.8-fold occurring between 14 and 21 days. At 21 days, dopamine levels reached $22.6 \mathrm{ng} / \mathrm{mg}$ of protein. Norepinephrine and serotonin levels were below the level of sensitivity of the assay in these co-aggregates.

Development of tyrosine hydroxylase. The estimated maximal velocity of tyrosine hydroxylase in RMT-CS coaggregates increased with time in culture (Fig. 3 ). There was a 2.3 -fold increase in activity between 3 and 14 days
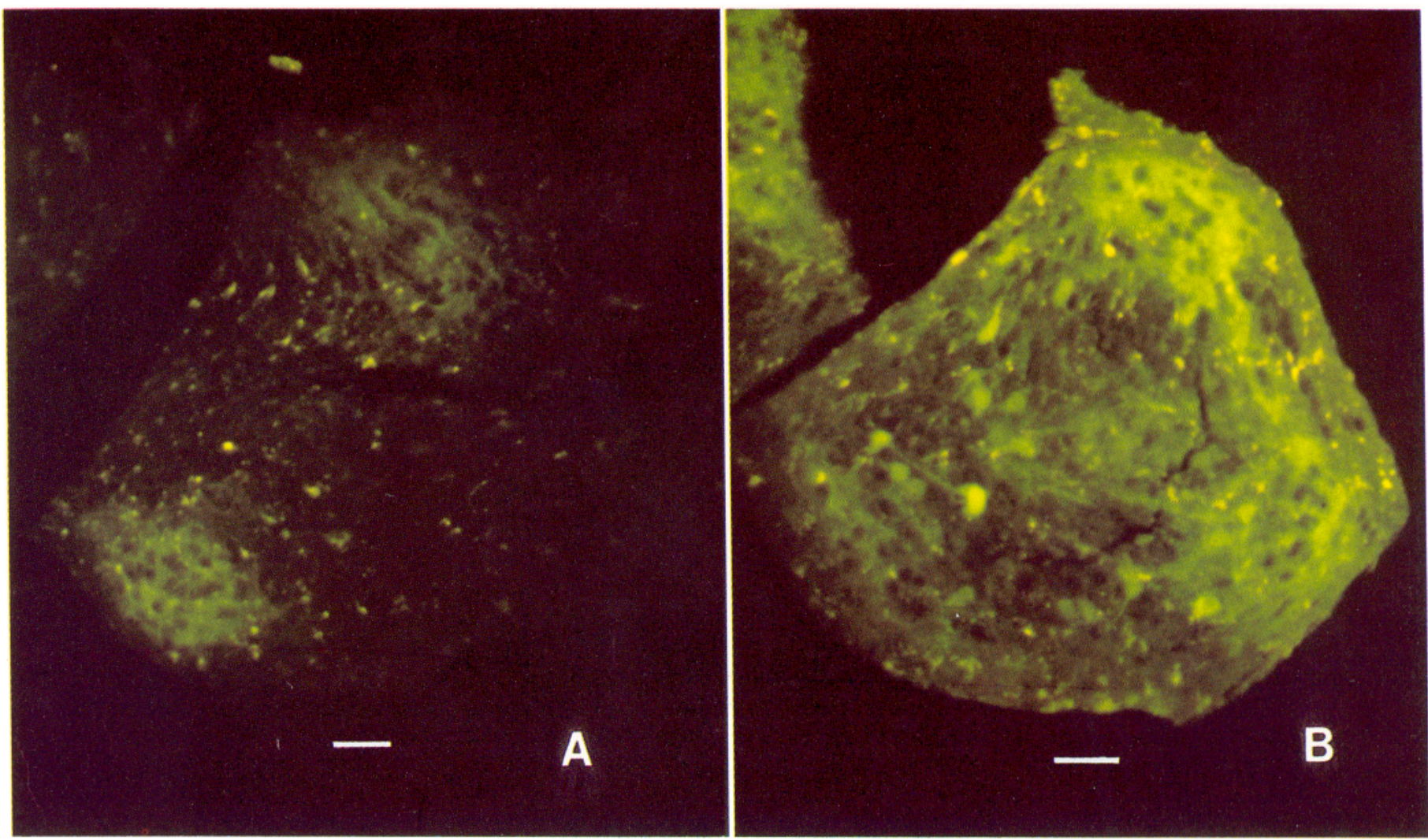

Figure 1. Endogenous dopamine histofluorescence in RMT-CS co-aggregates cultured for 14 and 21 days. A, A photomicrograph of a $10-\mu \mathrm{m}$ section through a 14-day RMT-CS co-aggregate processed by the Falck-Hillarp histofluorescence technique. Note the patches of punctate fluorescence. Scale bar, $30 \mu \mathrm{m}$. B, A photomicrograph of a 10- $\mu$ m section through a 21-day RMT-CS coaggregate processed by the Falck-Hillarp histofluorescence technique. Note the fluorescent cell bodies and patches of punctate fluorescence. Scale bar, $30 \mu \mathrm{m}$. 
in culture. However, the enzyme activity showed no further increment in 21-day-old cultures.

Development of the ability to accumulate exogenous dopamine as assessed by histofluorescence. After preincubation with $1 \mu \mathrm{M}$ dopamine, punctate pericellular fluorescence could be observed in 3- (Fig. 4) and 7-day-old co-aggregates which did not exhibit such fluorescence derived from endogenous dopamine. Treatment with exogenous dopamine resulted in enhanced fluorescence in 14-day-old co-aggregates. However, it was difficult to distinguish any increase due to the loading over the already very intense fluorescence present in the 21-day co-aggregates.

The punctate, pericellular fluorescence in the co-aggregates was absent if the RMT-CS co-aggregates had been pretreated with $100 \mathrm{nM}$ reserpine $24 \mathrm{hr}$ prior to loading with dopamine. However, fluorescent cell bodies could often still be observed although with diminished intensity. This loss of punctate pericellular fluorescence was particularly pronounced in the 14- and 21-day cultures in which nearly complete loss of the punctate pericellular fluorescence typical of untreated cultures was observed. Figures 5 and 6 are photomicrographs which accurately reflect the comparative loss of this fluorescence as seen with the microscope. Similar losses of punctate pericellular fluorescence were observed after pretreatment of the cultures with the neurotoxin 6OHDA $\left(10^{-4} \mathrm{M}\right)$, but in this case, no fluorescent cell bodies could be seen. The most dramatic losses again occurred in 14- and 21-day-old cultures.

Development of the capacity to accumulate exogenous dopamine as assessed by dopamine levels. Dopamine levels attained after the co-aggregates had been preincubated with $1 \mu \mathrm{M}$ dopamine and washed for $30 \mathrm{~min}$ after loading increased from $6.5 \pm 1.6 \mathrm{ng} / \mathrm{mg}$ of protein in 3 day cultures to $81.8 \pm 11.1 \mathrm{ng} / \mathrm{mg}$ of protein in 21 -day cultures (Fig. $7 A$ ). This pattern was maintained when co-aggregates were washed for $60 \mathrm{~min}$ after loading (Fig. $7 B$ ). These data also demonstrate that the co-aggregates retain 60 to $65 \%$ of the loaded dopamine despite an additional $30 \mathrm{~min}$ of washing. In these experiments, the

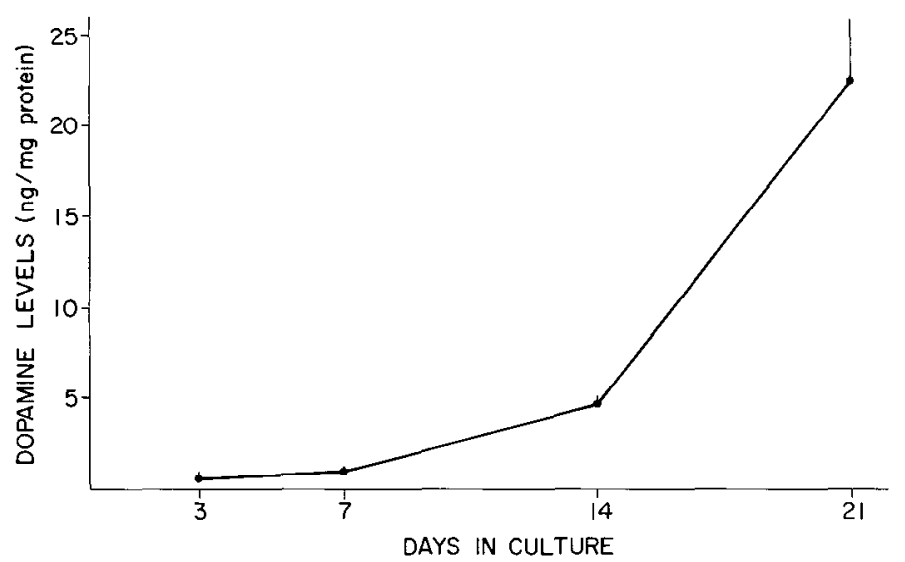

Figure 2. Development of levels of endogenous dopamine in RMT-CS co-aggregates. Details of the experimental procedures are given under "Materials and Methods." The data shown are the mean \pm SEM of three independent experiments. Each experiment involved analysis of the dopamine content in 23 flasks, each containing 90 to 120 aggregates.

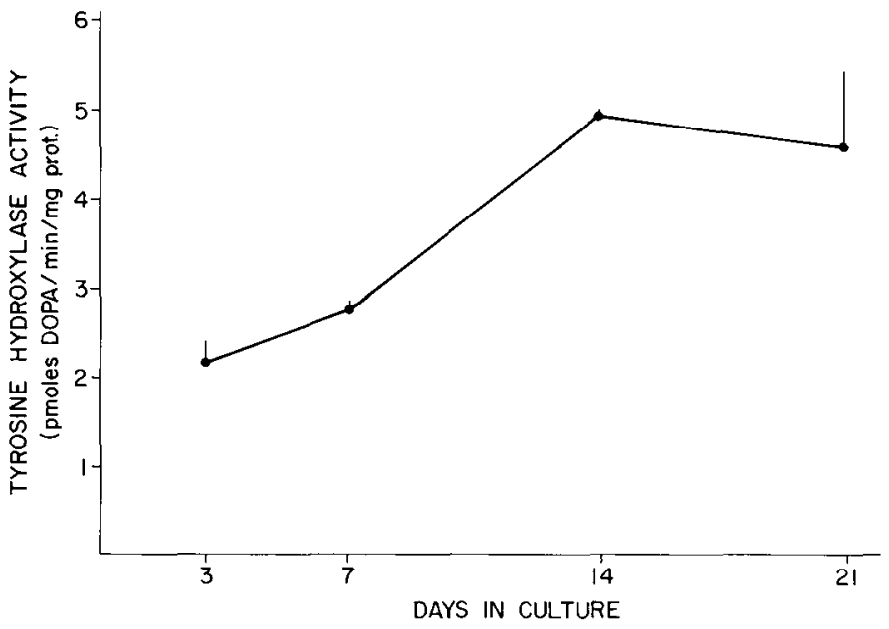

Figure 3. Development of tyrosine hydroxylase activity in RMT-CS co-aggregates. Details of the experimental procedures are included under "Materials and Methods." The data represent the mean \pm SEM for three independent experiments. Each experiment involved the analysis of the tyrosine hydroxylase activity of 1 flask containing 90 to 120 aggregates.

dopamine accumulated and retained increased from 4.5 $\pm 1.4 \mathrm{ng} / \mathrm{mg}$ of protein at 3 days to $52.6 \pm 5.6$ at 21 days in culture, an increase of 11.6 -fold (Fig. $7 B$ ). Pretreatment of RMT-CS cultures with reserpine (100 nM) $24 \mathrm{hr}$ prior to incubation with $1 \mu \mathrm{M}$ dopamine resulted in decreased accumulation of dopamine in 7-, 14-, and 21-day cultures while having no effect on 3-day cultures (Fig. $7 A$ ). The greatest effects of reserpine were observed in 14- and 21-day cultures. Cultures which had been treated with reserpine and washed for $30 \mathrm{~min}$ accumulate and retain only $5 \%$ and $5.7 \%$ as much dopamine, respectively, as untreated cultures. Virtually identical results were obtained in similar cultures washed for $60 \mathrm{~min}$ (Fig. $7 B$ ). Treatment with the neurotoxin 6-OHDA resulted in similar losses in the ability of aggregates to accumulate and retain dopamine at 7,14 , and 21 days of culture while leaving 3-day cultures unaffected. This agent is accumulated in catecholamine neurons by the specific amine uptake system and results in the destruction of catecholamine neurons (Kostrezwa and Jacobowitz, 1974). 6OHDA-treated co-aggregates cultured for 7,14 , and 21 days accumulated $41 \%, 10 \%$, and $7 \%$ of the dopamine accumulated by their respective vehicle-treated controls (Fig. 7, $A$ and $B$ ).

\section{Discussion}

The purpose of this investigation was to study the development and differentiation of dopamine neurons in RMT-CS co-aggregates in vitro utilizing morphological and biochemical techniques. Evaluation of the results obtained with the aggregating cell culture system requires consideration of the processes which occur during in vivo development and differentiation of dopamine neurons as well as the temporal patterns of these processes. The normal development of dopamine neurons is characterized by a number of events including (1) the birth and proliferation of dopamine cells, (2) the outgrowth of axonal and dendritic processes from the cell soma to appropriate terminal fields, $(3)$ the proliferation 


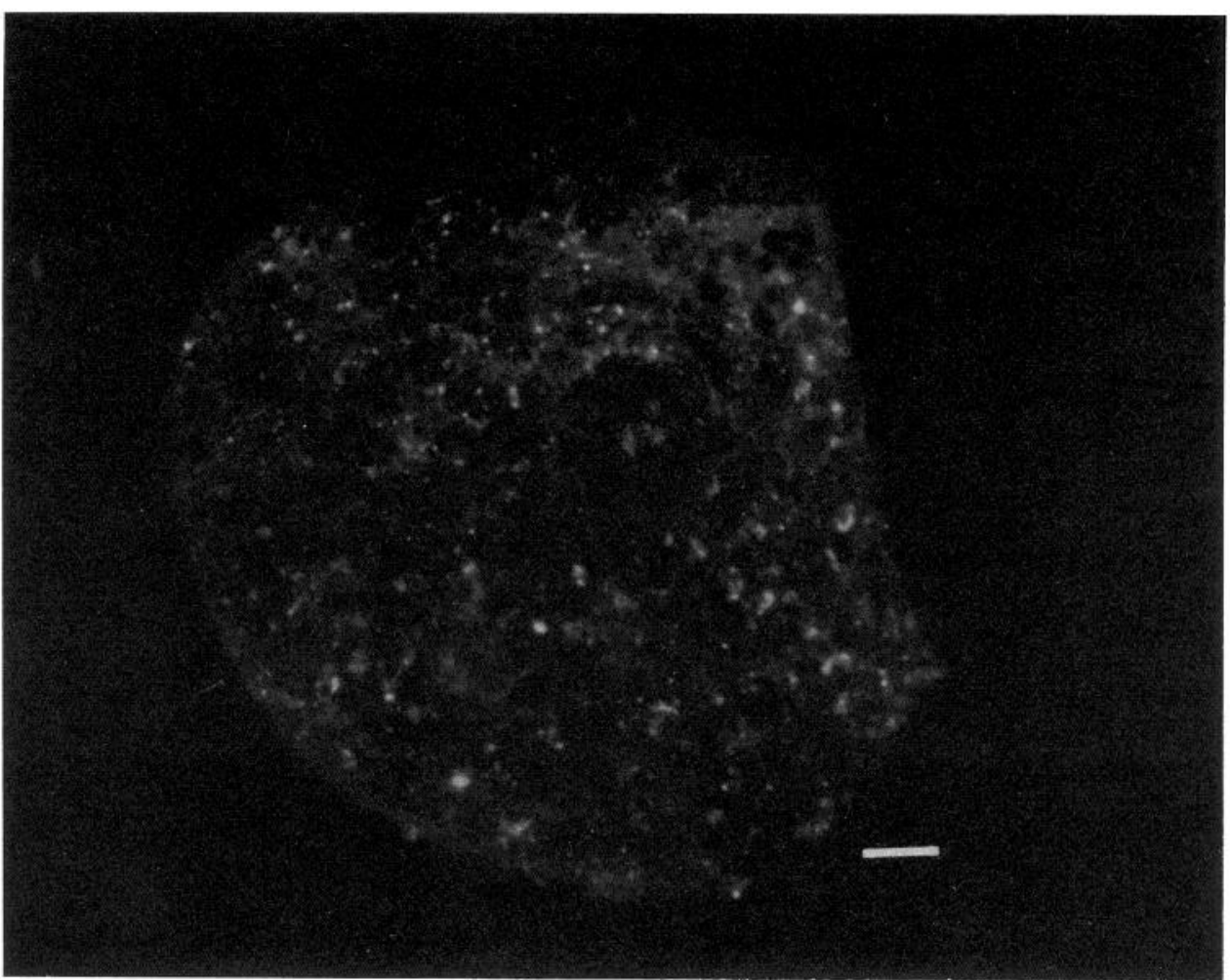

Figure 4. Dopamine histofluorescence in RMT-CS co-aggregates cultured for 3 days: a photomicrograph of a $10-\mu \mathrm{m}$ section through a 3-day RMT-CS co-aggregate incubated with $10^{-6} \mathrm{M}$ dopamine and processed by the Falck-Hillarp histofluorescence technique (see "Materials and Methods" for details). Note the small area of punctate fluorescence in the upper right corner. Scale bar, $30 \mu \mathrm{m}$.

of these processes, (4) the development of the enzymes necessary for the synthesis and metabolism of dopamine, and (5) the development of specific uptake and storage mechanisms for this neurotransmitter. Much of this differentiation is completed or is substantially underway during fetal life.

The birth and proliferation of dopamine neurons in the substantia nigra take place between the 12th and 14th day of gestation in the mouse as demonstrated by autoradiographic studies on the incorporation of $\left[{ }^{3} \mathrm{H}\right]$ thymidine (Coyle, 1974). The 14-day embryos used as the source of mesencephalic dopamine neurons in this study should, therefore, possess their full complement of dopamine neurons and these neurons should be predominantly postmitotic.

The outgrowth of dopaminergic axons and the proliferation of axonal arborization occur in vitro as shown by histofluorescent patterns previously observed in 7-day RMT-CS co-aggregates (Hemmendinger et al., 1981) and those observed in such co-aggregates at earlier and later time points in the present investigation. Previous studies in vivo have shown fluorescent catecholamine-containing processes coursing rostrally to the corpus striatum between the 13th and 16th day of gestation in the mouse (Golden, 1973). The proliferation of terminal processes occurs in late fetal and early postnatal life and essentially adult patterns of dopamine histofluorescence are observed by 28 days of age (Coyle, 1974; Olson and Seiger, 1972; Seiger and Olson, 1973). We have observed the appearance of punctate pericellular fluorescence as early as 3 days in culture (Fig. 4) when co-aggregates are preincubated with $1 \mu \mathrm{M}$ dopamine. This corresponds to a conceptual age of 17 days. The density of these patches of dopamine histofluorescence increases with time in culture (Figs. 4, 5A, and 6A). The fluorescent patterns observed in 21-day RMT-CS co-aggregates, irrespective of whether they have been pretreated with exogenous dopamine, are remarkably similar to the fluorescent patterns observed in the striatum of intact animals of an equivalent age (i.e., 35 days postconception). Therefore, it appears that the temporal course of development and the resulting pattern of dopaminergic axonal processes in the cultures parallel that seen in vivo over the time course examined.

Dopamine neurons in co-aggregates of RMT-CS also have an increasing ability to synthesize dopamine as they develop in culture as demonstrated by (1) the progressively increasing levels of endogenous dopamine measured over the 21-day culture period (Fig. 2), (2) the increasing activity of tyrosine hydroxylase (Fig. 3), and (3) the emergence of histofluorescence due to endogenous dopamine (Fig. 1).

Using immunocytochemical methods, tyrosine hydroxylase can be detected in neuronal perikarya at embryonic day 12.5 in the rat, immediately after these neurons have completed mitosis and migrated away from the proliferative ventricular layer (Specht et al., 1981). The ability to synthesize $\left[{ }^{3} \mathrm{H}\right]$ dopamine from $\left[{ }^{3} \mathrm{H}\right]$ tyrosine has been 

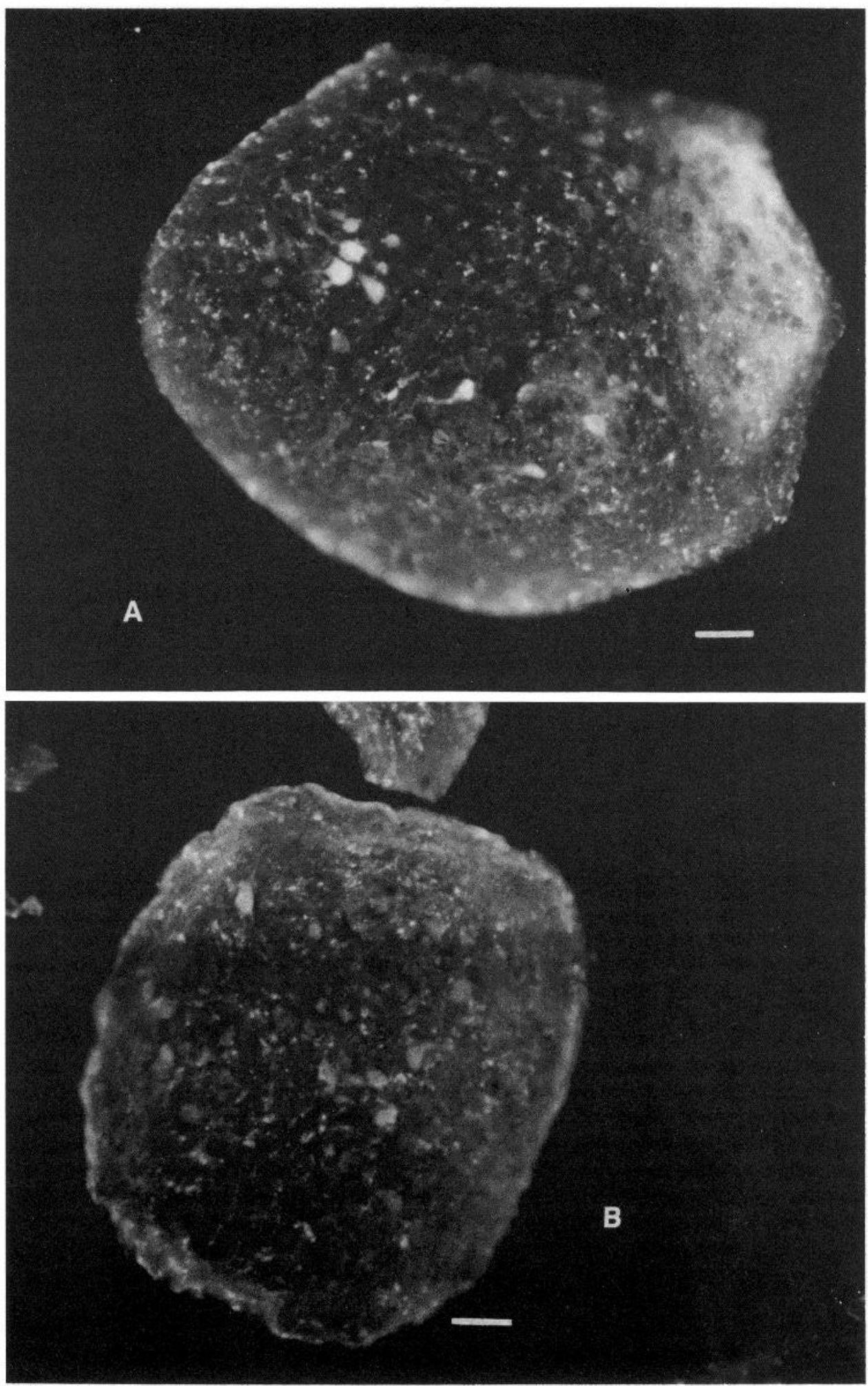

Figure 5. Dopamine histofluorescence in RMT-CS co-aggregates cultured for 14 days: effect of reserpine. $A$, A photomicrograph of a $10-\mu \mathrm{m}$ section through a 14-day RMT-CS co-aggregate incubated with $10^{-6} \mathrm{M}$ dopamine prior to processing by the Falck-Hillarp histofluorescence technique. Note the areas of punctate fluorescence and fluorescent cell bodies. Scale bar, $30 \mu \mathrm{m}$. B, A photomicrograph of a $10-\mu \mathrm{m}$ section through a 14-day RMTCS co-aggregate pretreated with $10^{-7} \mathrm{M}$ reserpine and incubated with $10^{-6} \mathrm{M}$ dopamine prior to processing by the Falck-Hillarp histofluorescence technique. Note the absence of punctate fluorescence. Scale bar, $30 \mu \mathrm{m}$. 

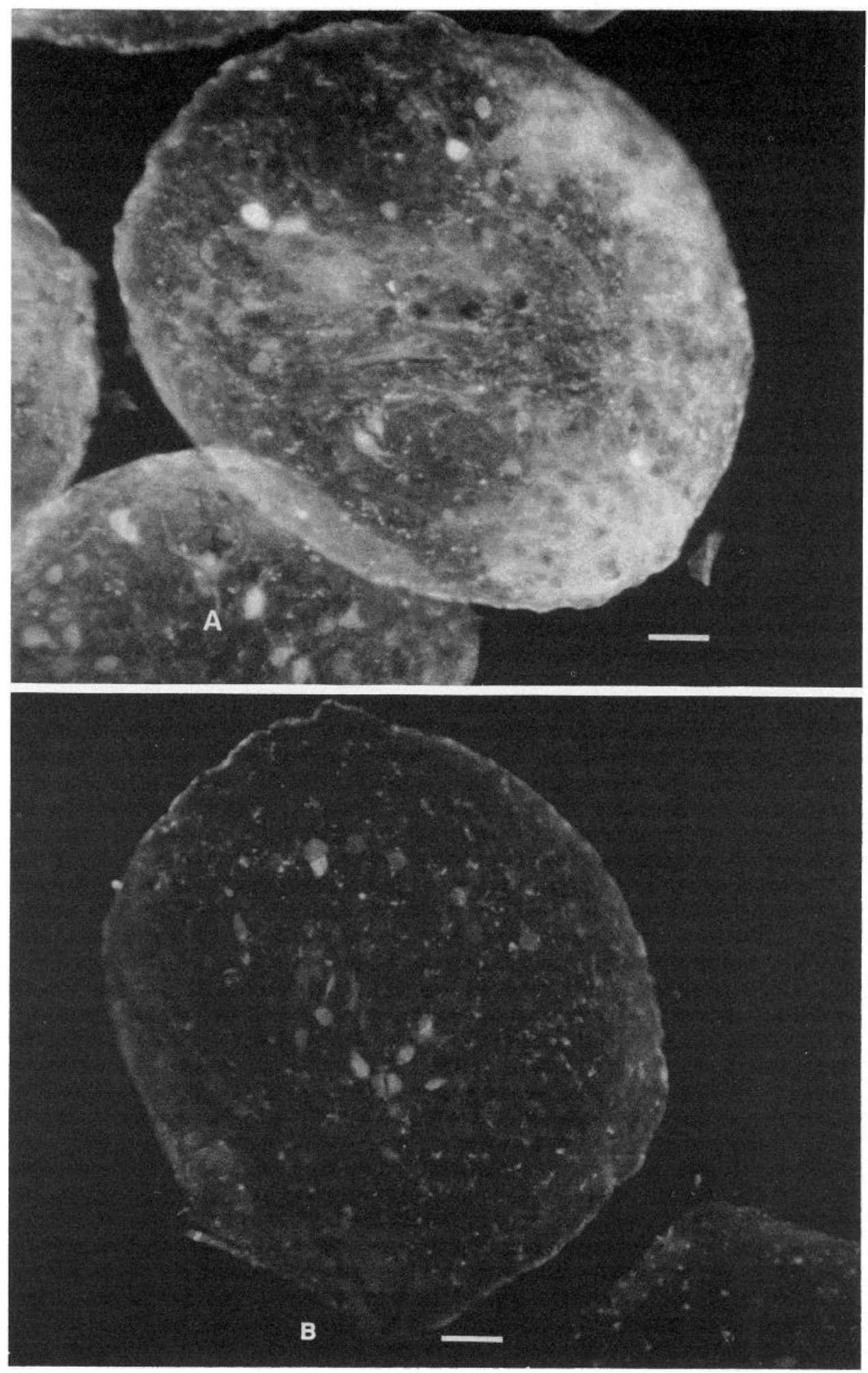

Figure 6. Dopamine histofluorescence in RMT-CS co-aggregates cultured for 21 days: effect of reserpine. $A$, A photomicrograph of a $10-\mu \mathrm{m}$ section through a 21 -day RMT-CS co-aggregate incubated with $10^{-6} \mathrm{M}$ dopamine prior to processing by the Falck-Hillarp histofluorescence technique. Note the areas of punctate fluorescence and fluorescent cell bodies. Scale bar, $30 \mu \mathrm{m}$. B, A photomicrograph of a 10- $\mu \mathrm{m}$ section through a 21-day RMTCS co-aggregate pretreated with $10^{-7} \mathrm{M}$ reserpine and incubated with $10^{-6} \mathrm{M}$ dopamine prior to processing by the Falck-Hillarp histofluorescence technique. Note the absence of punctate fluorescence. Scale bar, $30 \mu \mathrm{m}$. 


\section{Control $\square \quad$ Reserpine $\square \quad 6$-OHDA $[\mathrm{m}$}
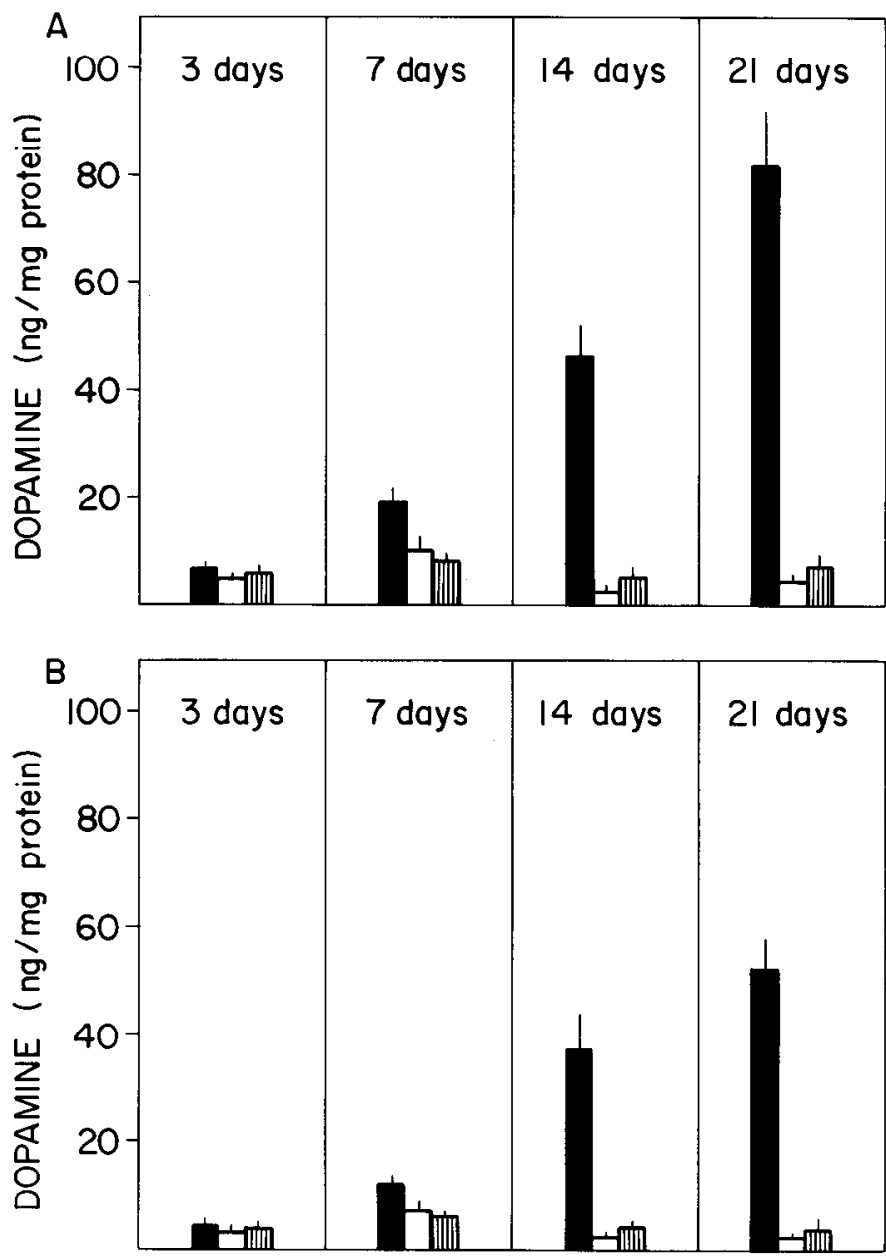

Figure 7 . Development of the capacity for dopamine accumulation in RMT-CS co-aggregates: effect of reserpine and 6OHDA. The data shown in the upper panels $(A)$ represent the dopamine levels of RMT-CS co-aggregates loaded with $10^{-6} \mathrm{M}$ dopamine for $10 \mathrm{~min}$ followed by a 30 -min incubation in dopamine-free medium. The data shown in the lower panels $(B)$ represent the dopamine levels of RMT-CS co-aggregates loaded with $10^{-6} \mathrm{M}$ dopamine followed by a $60-\mathrm{min}$ incubation in dopamine-free medium. Experimental details are given under "Materials and Methods." The data shown are the mean \pm SEM of three or four independent experiments.

reported in aggregating cell cultures formed from dissociated cells of whole rat brain or the midbrain plus hindbrain of 15-day-old rat embryos (Honegger and Richelson, 1979). $\left[{ }^{3} \mathrm{H}\right]$ Dopamine synthesis also has been reported to occur in monolayer cultures of RMT neurons (Prochiantz et al., 1979) although this activily was not dependent on culture age.

The estimated maximal velocity of tyrosine hydroxylase increases in the reaggregate culture system between days 3 and 14 in culture and remains elevated at 21 days (Fig. 3). These results are somewhat surprising in that no further increase in activity was observed between 14 and 21 days, since lyrosine hydroxylase levels in vivo increase 12-fold during this period (Coyle, 1974). This deviation from the in vivo developmental pattern may be the result of changes in kinetic properties for the enzyme during the period of 14 to 21 days. Such changes would not have been revealed in our experiments since we examined enzymatic activity at a single concentration of substrate and cofactor and at a single incubation time. Resolution of this issue requires a more thorough analysis of the kinetic properties of this enzyme in cultured reaggregates at various stages of development. In this regard, it should be noted that the maximal tyrosine hydroxylase activity in 21-day aggregates is considerably less than that obtained in mechanically dissociated cell-reaggregating cultures of rat mesencephalon and diencephalon (Honegger and Richelson, 1977). Given the experimental differences in the two investigations, it is difficult to make direct comparisons between the two sets of data.

An alternative explanation for the failure of tyrosine hydroxylase activity to increase between 14 and 21 days is that the proliferation of axonal processes, and consequently the amount of tyrosine hydroxylase in the coaggregates, is dependent on the number of target cells available to the dopamine neurons of the RMT. Since, in the co-aggregates, there are greater numbers of RMT cells relative to CS cells than there are in vivo, axonal processes may continue to proliferate in vivo over a longer time course than is required to innervate the CS cells fully in vitro. This alternative can be examined experimentally by growing RMT-CS co-aggregates with fewer numbers of RMT cells relative to the CS cells and examining the time course of the development of tyrosine hydroxylase activity.

RMT-CS co-aggregates also have the ability to accumulate and retain exogenous dopamine. This represents the net result of both the processes involved in specific amine uptake by dopamine neurons and those involved in the storage of amines by these neurons. The development of specific amine uptake and vesicular storage of amines parallels the proliferation of dopamine terminals in vivo (Coyle, 1974). The present experiments show that there is a low, but detectable, accumulation of dopamine in the reaggregate culture system as early as 3 days in culture which subsequently increases 11 - to 13 -fold by 21 days in culture (Fig. 7). This accumulation of dopamine is apparently specific to dopamine neurons since norepinephrine and serotonin are not detectable in these coaggregates, implying the absence of noradrenergic and serotonergic neurons. This conclusion also is supported by studies with specific inhibitors of the uptake process (Hemmendinger et al., 1981).

6-OHDA must be accumulated in neurons for it to exert its neurotoxic effect (Kostrezwa and Jacobowitz, 1974). The relative inability of 3-day cultures to accumulate and retain dopamine may explain the insensitivity of these processes to 6-OHDA at this age, since the neurons also may accumulate insufficient 6-OHDA to result in toxicity.

RMT-CS co-aggregates also develop a vesicular storage capacity in vitro as demonstrated by the effects of reserpine treatment on co-aggregates of various culture ages. Reserpine has been shown to disrupt the vesicles contained in catecholaminergic neurons which form the major storage sites of norepinephrine and dopamine in vivo. The effects of reserpine treatment also have been observed early in ontogeny (i.e., 18 days gestation; Coyle, 
1974). Co-aggregates treated with $100 \mathrm{~nm}$ reserpine at 7 , 14 , or 21 days in culture showed a profound loss in the ability to retain exogenous dopamine compared to untreated co-aggregates which could retain dopamine for periods up to $60 \mathrm{~min}$ when placed in dopamine-free media. In contrast to its effect on more mature cultures, reserpine treatment of 3-day cultures produced only small decreases in the amount of dopamine accumulated by the dopaminergic neurons. This may be the result of functional immaturity or a low number of storage vesicles in these younger cultures. Further studies in which the processes of amine uptake and vesicular storage are studied separately will be necessary to elucidate the relative contributions of these processes and the details of their ontogeny.

These experiments have demonstrated that dopamine neurons develop a number of characteristics in the in vitro dissociated cell-reaggregate culture system which are typical of dopamine neurons in vivo. Specifically, they devclop an increasing ability to synthesize, accumulate, and store dopamine while the neurons are elaborating and maintaining axonal processes. Furthermore, the pharmacologic characteristics of these processes are similar to those observed in vivo. The developmental course of these functions is also consistent with that which would be expected from observations on the developmental course that these processes follow in vivo. This in vitro culture system, therefore, represents a useful model system for studying the mechanisms which direct the development and differentiation of dopamine neurons since it permits experimental control of both the cellular and fluid environment of the dopamine neurons during their development.

\section{References}

Coyle, J. T. (1974) Development of central catecholaminergic neurons. In The Neurosciences: Third Study Program, F. O. Schmitt and F. G. Worden, eds., pp. 877-884, MIT Press, Cambridge, MA.

Coyle, J. T., and P. Campochiaro (1976) Ontogenesis of dopaminergic-cholinergic interactions in the rat striatum. J. Neurochem. 27: 673-678.

Falck, R., N. -A. Hillarp, G. Thieme, and A. Torp (1962) Fluorescence of catecholamines and related compounds condensed with formaldehyde. J. Histochem. Cytochem. 10: 348-354.

Garber, B. B., and A. A. Moscona (1972) Reconstruction of brain tissue from cell suspensions. I. Aggregation patterns of cells dissociated from different regions of the developing brain. Dev. Biol. 27: 217-234.

Golden, G. S. (1973) Prenatal development of the biogenic amine systems of the mouse brain. Dev. Biol. 33: 300-311.

Hemmendinger, L. M., B. B. Garber, P. C. Hoffmann, and A. Heller (1981) Target neuron-specific process formation by embryonic mesencephalic dopamine neurons in vitro. Proc. Natl. Acad. Sci. U. S. A. 78: 1264-1268.

Honegger, P., and F. Richelson (1977) Biochemical differentiation of aggregating cell cultures of different fetal rat brain regions. Brain Res. 133: 329-339.

Honegger, P., and E. Richelson (1979) Neurotransmitter synthesis, storage and release by aggregating cell cultures of rat brain. Brain Res. 162: 89-107.

Kostrezwa, R. M., and D. M. Jacobowitz (1974) Pharmacological actions of 6-hydroxydopamine. Pharmacol. Rev. 26: 199-288.

Lauder, J. M., and F. E. Bloom (1974) Ontogeny of monoamine neurons in the locus coeruleus, raphe nuclei and substantia nigra of the rat. I. Cell differentiation. J. Comp. Neurol. 155: 469-482.

Lowry, O. H., N. J. Rosebrough, A. L. Farr, and R. J. Randall (1951) Protein measurement with the Folin phenol reagent. J. Biol. Chem. 193: 265-275.

Moore, R. Y., and F. E. Bloom (1978) Central catecholamine neuron systems: Anatomy and physiology of the dopamine systems. Annu. Rev. Neursoci. 1: 129-169.

Olson, L., and A. Seiger (1972) Early prenatal ontogeny of central monoamine neurons in the rat: Fluorescence histochemical observations. Z. Anat. Entwicklungsgesch. 137: 301-316.

Porcher, W., and A. Heller (1972) Regional development of catecholamine biosynthesis in rat brain. J. Neurochem. 19: 1917-1930.

Prochiantz, A., U. DiPorzio, A. Kato, B. Berger, and J. Glowinski (1979) In vitro maturation of mesencephalic dopaminergic neurons from mouse embryos is enhanced in the presence of their striatal target cells. Proc. Natl. Acad. Sci. U. S. A. 76: 5387-5391.

Seiden, L. S., and L. A. Dykstra (1977) Dopamine, norepinephrine and behavior. In Psychopharmacology: A Behavioral and Biochemical Approach, pp. 117-171, Van Nostrand Reinhold Co., New York.

Seiger, A., and L. Olson (1973) Late prenatal ontogeny of central monoamine neurons in the rat. Fluorescence histochemical observations. Z. Anat. Entwicklungsgesch. 410: 281-318.

Specht, L. A., V. M. Pickel, T. H. Joh, and D. J. Reis (1981) Light microscopic immunocytochemical localization of tyrosine hydroxylase in prenatal rat brain. I. Early ontogeny. J. Comp. Neurol. 199: 233-253.

Ungerstedt, U. (1971) Stereotaxic mapping of the monoamine pathways in the rat brain. Acta Physiol. Scand. 367: 1-48. 